\title{
Grand challenges in plant physiology: the underpinning of translational research
}

\author{
Steven C. Huber ${ }^{1,2 *}$ \\ 1 United States Department of Agriculture-Agricultural Research Service, University of Illinois, Urbana-Champaign, IL, USA \\ 2 Departments of Plant Biology and Crop Science, University of Illinois, Urbana-Champaign, IL, USA \\ *Correspondence: schuber1@illinois.edu
}

A grand challenge, it would seem, has to be grand, i.e., large and impressive in size, scope, or extent. While each plant physiologist would likely come up with a list of challenges in their own area of work, to be truly grand these should span the entire discipline of plant physiology, and ideally have significant impact on both science and technology. The question then is what is the scope of "plant physiology?" Turns out there are numerous definitions in use. This journal considers it to encompass the "nature of functioning of an organism" studied at various levels of complexity and embracing the full range of technologies from cell biology to systems biology. Likewise, the journal with this name considers it to include broad aspects of plant biology, including an "understanding of the plant as a whole organism and its interactions with symbionts, pathogens and pests, and the environment," and employing disciplines ranging from cell and molecular biology, biochemistry, and biophysics to genetics and physiology (Chrispeels and Raikhel, 2000). These are consonant with my definition of plant physiology, which I consider to be generally synonymous with plant science and plant biology. Thus, I see "physiology" as a relevant component of most if not all of the Frontiers specialties listed on the journal website ${ }^{1}$ and believe it would be difficult to come up with a definition of plant physiology that excluded these areas. However, in the challenges discussed in this article I will try to minimize the overlap with the challenges elaborated in other Specialities. A corollary to this is that future authors should recognize that articles destined for the Frontiers in Plant Science series could fit under several Specialities, and they are free to decide where their work would fit best.

${ }^{1}$ www.frontiersin.org/plant_physiology
In my view, the truly grand challenges in plant physiology are those that will yield important insights about how plants live and function, and also provide at least partial solutions to critical societal needs. Some of the most pressing needs are contained in the recent A New Biology report from the National Research Council (NRC, 2009), which outlined sustainable food production, ecosystem restoration, biofuel production, and human health as four major societal needs - grand challenges indeed. These same challenges show up in the areas targeted by the recently created National Institute for Food and Agriculture (NIFA) that has focused competitive research programs around the themes of climate change; bioenergy; food safety; nutrition and childhood obesity; and global food security. Similarly, the recently formed Global Plant Council has identified world hunger, energy, climate change, health and well-being, sustainability and environmental protection as major challenges that need to be addressed on a broad scale ${ }^{2}$. Thus, I would submit that the grand challenge is to determine how plant physiology research can be applied to help solve these major societal needs, which will likely involve integrated multidisciplinary approaches with physiology playing an important role.

So how can plant physiology research help achieve the grand challenge? Taking a fairly restrictive view of what constitutes physiology research, we might consider each of the three traditional areas of physiology research: (i) metabolism (including nutrition); (ii) growth and development (vegetative and reproductive); and (iii) response to the environment. Each of these is an area of active work where breakthroughs could result in progress toward societal needs,

${ }^{2} \mathrm{http} / / /$ globalplantcouncil.org and a partial list (and admittedly biased) of specific challenges and opportunities is highlighted below.

\section{METABOLISM AND NUTRITION}

In the broad area of metabolism, we continue to learn important things about pathways, including the role played by different enzymes and gene products. For example, the sucrose synthases (SUSs) and invertases are the two enzyme classes that catalyze sucrose catabolism in plants. This may sound like a specific step in a dull "housekeeping" pathway, but is extremely important as the majority of plant material that we (and other animals) eat is formed from the sucrose metabolized by one of these two enzymes! It had long been thought that SUS was the universally important enzyme of the two, because of the critical role this enzyme plays in production of starch in developing cereals. However, recent work in Alison Smith's laboratory using T-DNA insertional mutants (knockouts) of SUS genes in Arabidopsis revealed that under normal growth conditions, the SUS genes were essentially dispensable and (surprisingly) neutral cytosolic invertase emerged as the generally essential enzyme (Barratt et al., 2009). An understanding of pathway components is essential if we are to manipulate or engineer them to address societal challenges, and clearly there is much to learn.

To this end, pathways involved in photosynthesis, respiration, cell wall biosynthesis, and biological nitrogen fixation are being evaluated with an eye to manipulate them or to transfer unique aspects among species. Much of this work is driven by anticipated increases in global demand for food and bioenergy in the near future. For example, photosynthesis is being targeted for manipulation in several ways. One ambitious goal is to enhance the rate of $\mathrm{CO}_{2}$ assimilation (and hence increase yield) in a crop plant such as rice by incorporation 
of the more efficient $\mathrm{C}_{4}$-pathway to yield " $\mathrm{C}_{4}$ rice" (Hibberd et al., 2008; Hibberd and Covshoff, 2010). Alternatively, photosynthesis in $\mathrm{C}_{3}$ crops such as rice, wheat, and soybean may be enhanced in the future by improving solar energy conversion efficiency; e.g., by incorporating chlorophyll molecules that can absorb light in the near-infrared range that is currently not absorbed and thereby increase the fraction of solar energy utilized (Blankenship et al., 2011). More radical alterations in the photosystems that drive electron transport can also be envisaged and might include incorporating photovoltaic cells or switching plants from carbon reduction to $\mathrm{H}_{2}$ production (Blankenship et al., 2011). Dramatic alterations of this sort would likely involve synthetic biology approaches (Haseloff and Ajioka, 2009). The flip side of photosynthesis is respiration and the two processes are intimately connected. Respiration involves processes associated with growth and maintenance and collectively is a key determinant of biomass production and yield. Much remains to be learned about the levels at which respiration is controlled; e.g., how respiratory activity is impacted by environmental conditions (Millar et al., 2011). Along similar lines, efforts to transfer the process of biological nitrogen fixation found in legumes to non-leguminous crops such as cereals, could dramatically reduce the fertilizer inputs needed for the intense agricultural systems of the future. As envisioned (Charpentier and Oldroyd, 2010), this would involve a series of engineering steps that essentially mimic the evolutionary processes that occurred in legumes, but alternatively, synthetic biology approaches might one day also be employed to achieve this dramatic change.

A greater understanding of cell wall biosynthesis and manipulation is another broad area that could impact societal needs in the future. While much is already known about the complexities of cell wall production, much remains to be learned as well (Popper et al., 2011). Moreover, in order for plant cell walls to be utilized as second generation biofuels, more efficient means of "deconstruction" of cell wall polymers must be achieved (Himmel et al., 2007), and will almost certainly involve genetic modification of cell wall constituents (Fu et al., 2011).
If metabolic pathways are to be manipulated, it is equally important (in fact essential) to understand how the pathways are regulated. Pathway regulation can occur at multiple levels including (broadly) the transcriptional, translational, and posttranslational levels, and much remains to be learned about the fundamentals of each process and how they collectively contribute to pathway regulation. Relative contributions of the different levels will certainly vary depending on the stimulus. Rapid changes in flux often involve regulation at the protein level, but we still lack comprehensive information about the structure-function relationships of proteins (Zwieniecki and Dumais, 2011) and the various reversible modifications that proteins can undergo at the posttranslational level. Understanding the diversity of posttranslational modifications (PTMs) is identified as a critical need area that includes an appreciation of which modifications of metabolic pathways occur in vivo and are of potential regulatory importance. The importance and inherent difficulties associated with identifying PTMs of proteins is also recognized as a major challenge in plant proteomics (Heazlewood, 2011).

Protein phosphorylation is generally considered to be the most abundant PTM in eukaryotes, and the robust nature of phosphorylation in plants is being documented with large-scale phosphoproteomic screening. While more than 30,000 phosphosites have been identified in these studies (as compiled by the Arabidopsis Protein Phosphorylation Site Database, $\mathrm{Phos}_{\mathrm{PhAt}}{ }^{3}$ ) there is a broad need for more quantitative analyses and determination of the functional significance of these sites. The former approaches are often high throughput, while the latter are typically low throughput as moving from correlation analysis to establishing cause-and-effect relationships often involves directed mutagenesis and targeted functional analyses. What has been established is that in plants as in animals, the occurrence of phosphorylation on serine, threonine, and tyrosine residues is roughly 75:20:5 (Sugiyama et al., 2008). The prevalence of tyrosine phosphorylation (discussed further below) was surprising because plants seem to lack traditional

${ }^{3}$ http://phosphat.mpimp-golm-gdm.mpg.de/ tyrosine kinases and thus, identifying the protein kinases involved remains a challenge.

Phosphorylation is just one of the many possible PTMs in vivo that results in proteome diversification, and research continues to reveal the occurrence of new PTMs that may regulate key metabolic steps. For example, mono-ubiquitination has recently been identified to occur with the enzyme phosphoenolpyruvate carboxylase (PEPC; Uhrig et al., 2008). Mono-ubiquitination is a reversible modification that promotes interaction of the modified protein with ubiquitin-binding domain proteins and thus is distinct from poly ubiquitination that targets proteins for proteasome degradation. Regulation of protein:protein interactions by mono-ubiquitination is an emerging area in plants and animals (Mukhopadhyay and Riezman, 2007) and likely plays a broader role in regulation of plant metabolism that is currently recognized. On a similar note, lysine acetylation (LysAc) has recently been found to be a robust modification of numerous proteins in Arabidopsis in addition to the histone proteins and in cellular compartments outside of the nucleus (Finkemeier et al., 2011; Wu et al., 2011). A number of metabolic enzymes were found to be acetylated, including ribulose-1,5-bisphosphate carboxylase/oxygenase (Rubisco) and Rubisco activase, and preliminary evidence suggests that acetylation may inhibit carboxylase activity (Finkemeier et al., 2011), making these modifications targets for manipulation that may impact plant productivity. It was thought for a long time that LysAc was a histone-specific modification that was restricted to the nucleus, but it is clear now that in plants, as in animals, and microbial systems (Glozak et al., 2005; Kim et al., 2006; Wang et al., 2010) that is not the case. Lysine acetylation could have direct effects on enzyme activity and could also be interactive with other modifications where Lys residues play a role; e.g., ubiquitination and phosphorylation (which can involve Lys residues as recognition elements in phosphorylation motifs). Another unusual modification that warrants mention is methionine (Met) oxidation. It is well known that Met residues on the surface of proteins (i.e., solvent exposed) can be very susceptible to oxidation to Met sulfoxide (MetSO), and has traditionally been considered to be oxidative damage 
and an unavoidable consequence of aerobic metabolism. Another view is that the propensity for Met residues to oxidize has been exploited in nature in some cases, at least, to serve another function - as a redox switch impacting other functions or modifications of the protein and thereby functioning as a regulatory PTM. One emerging example of how the "Met redox switch" could impact another modification is its interaction with phosphorylation of nearby residues (Hardin et al., 2009). When the Met residue in question functions as an essential recognition element in a phosphorylation motif, its oxidation can strongly inhibit phosphorylation of the targeted serine or threonine. However, a critical remaining question is whether this occurs in vivo, and to test this unequivocally will require studies with transgenic plants expressing directed mutants. These selected examples highlight the fact that much remains to be learned about metabolic pathways and their mechanisms of regulation. You can't translate what you don't understand!

\section{GROWTH AND DEVELOPMENT}

Growth and development is another traditional component of plant physiology that minimally includes processes such as cell division/elongation, photomorphogenesis and tropisms, endogenous clocks, and hormone systems. Each is an active area of research and new insights are being revealed daily. Within the area of hormone biology, pathways of signal transduction have now been generally elucidated for each of the major hormones starting from perception and resulting in changes in gene expression. However, how signaling is regulated and integrated across multiple hormones to control plant growth remains a major challenge. Moreover, new plant growth regulators almost undoubtedly await discovery. In terms of mode of action, three of the hormones (auxin, jasmonates, and strigolactone) involve regulated proteolysis and because plants contain very large families of F-box proteins (involved in targeted proteolysis) it has been speculated that ligand-regulated proteolysis may be much more extensive than currently recognized (Somers and Fujiwara, 2009). Searching for new plant growth regulators and identifying their receptors and associated signaling pathways is clearly a major challenge for the future. Likewise, with each of the known hormones much remains to be done. As one example, the brassinosteroids (BRs) are essential growth-promoting hormones that are ligands for the leucine-rich repeat, receptor-like kinase (LRR-RLK) known as BRASSINOSTEROID INSENSITIVE 1 (BRI1). The signaling mechanisms elucidated for BRI1 and its co-receptor kinase, BRI1 ASSOCIATED KINASE 1 (BAK1), may provide a model for the $\sim 220$ other members of the LRR-RLK gene family in Arabidopsis - the majority of which have no recognized function. Identifying the ligands for these "orphan receptors" and the signaling pathways they presumably function in is also an extremely important area for future work and may provide unanticipated opportunities to engineer crop plants. To further consider BR signaling, the potential for improvement of agriculture has long been recognized since BRs generally enhance growth (vegetative and reproductive) and increase stress tolerance. Although somewhat counterintuitive, a weak loss of function mutant of rice BRI1 resulted in semi-dwarf plants with more erect leaves and thus increased potential for grain yield when planted at high density as in the field (Morinaka et al., 2006). However, a distinct and important challenge is to utilize the detailed knowledge of BR signaling to specifically engineer the system to increase plant productivity (Kim and Wang, 2010). One small step in this direction has just been achieved by altering the ability of BRI1 to autophosphorylate on a specific tyrosine residue (Tyr-831). The function of phosphorylation at the Tyr-831 site appears to be to attenuate BR signaling, and preventing this modification enhances BR signaling and increases Arabidopsis shoot growth (Oh et al., 2011). An obvious and critical next step is to determine whether these responses translate to crop plants.

The physiology of growth and development also includes reproductive stages, of course, about which much remains to be learned. For example, the underlying mechanisms that regulate seed composition are not fully understood and this has relevance to our use of harvested seeds as food and fuel, and also has potential relevance to biotechnology. A specific example of the latter notion involves soybean seeds, which normally accumulate protein and oil as major storage products in protein storage bodies and oil bodies, respectively. Fundamental studies of the function of the endomembrane system of seeds in formation of oil bodies and storage vacuoles (Schmidt and Herman, 2008) combined with genetic engineering is providing the foundation for novel approaches to engineer seeds to produce high levels of foreign proteins (of pharmaceutical or industrial interest) instead of the normal storage proteins (Boothe et al., 2010). Thus, fundamental physiology/cell biology research is providing the foundation for biotechnology innovations that have practical applications and potential for helping to meet societal needs in the future.

\section{RESPONSES TO THE ENVIRONMENT}

The third traditional component of plant physiology involves, in broad terms, plant responses to the environment. This can include stress physiology (both biotic and abiotic stress) and the broad topic of "climate change" impact on plant function. Thus, physiology plays an important role in functional plant ecology (Koerner, 2011) and plant-microbe interactions, both of which are also separate Specialities under the Frontiers in Plant Science umbrella. Understanding how crop plants respond to climate change is particularly important because agriculture is extremely sensitive to climate. Environmental factors involved in climate change that are expected to have the greatest impact on plant physiology include increased atmospheric $\left[\mathrm{CO}_{2}\right]$ and $\left[\mathrm{O}_{3}\right]$, temperature, and variability in climate (temperature extremes and changes in rainfall patterns). Of particular interest is to what extent the increase in atmospheric $\left[\mathrm{CO}_{2}\right]$ will offset the predicted rise in $\left[\mathrm{O}_{3}\right]$ and decreased soil moisture that is predicted for some regions. Interestingly, the impact of the " $\mathrm{CO}_{2}$ fertilization effect" tends to be greater from studies in greenhouses, growth chambers, and transparent field chambers compared to free-air concentration enrichment (FACE) technology that uses fully open-air field conditions and more accurately mimics the real-world environment (Long et al., 2006). However, regardless of the magnitude of the fertilization effect, understanding how plants respond to climate change conditions, including extremes of temperature and water, will be an essential aspect of plant physiology research in the future and identifying genes that confer stress tolerance is essential (Varshney et al., 
2011). It will also be important to conduct more controlled experiments where different stresses are applied simultaneously. For example, plants respond differently to heat and drought stress that are applied together compared to the single stresses (Rizhsky et al., 2004). In all of these approaches, high throughput phenotyping techniques to measure those traits and identify underlying genes will almost certainly play an important role (Berger et al., 2010). At the population level, determining how predicted changes in climate will impact crop growth and crop yield will remain an important area of research.

Climate change may also affect biotic stress factors for which the climate change is favorable and it appears that biotic stress may generally increase in the future. For example, for reasons that are not entirely clear, elevated atmospheric $\left[\mathrm{CO}_{2}\right]$ tends to reduce soybean defenses against herbivory (Zavala et al., 2008). How sensitivity to other plant pests such as phytopathogenic bacteria may be affected is not clear but may at least in some cases be increased in severity as well. Accordingly it is essential to understand the mechanisms that underlie plant detection of pests and allow them to mount defenses and how those mechanisms are impacted by environment, and ideally then use that information to increase pest resistance in crop plants. Invading microorganisms are often detected when plants recognize conserved molecules containing pathogen-associated molecular patterns (known as PAMPs) and initiate defense mechanisms that increase plant resistance to the infection. There are many fundamental questions that remain to be fully answered. For example, what are the potential PAMPs and how are they perceived? What is the role of PAMP-triggered immunity (PTI) in plant immunity? And most importantly, can disease resistance be increased by manipulating the system? One of the best studied mechanisms involves perception of PAMPs by pattern-recognition receptors (PRRs), many of which are LRR-RLKs that initiate signaling pathways resulting in PTI. One very exciting development in this area involves the transfer of a PRR gene from one species to another to confer responsiveness to novel PAMPs not normally recognized. In Arabidopsis, bacterial elongation factor $\mathrm{Tu}$ (EF-Tu) is recognized as a PAMP by the receptor kinase EFR, but EF-Tu is not recognized in the solanaceous plants Nicotiana benthamiana or Solanum lycopersicon. Expression of Arabidopsis EFR in both solanaceous species allows them to recognize bacterial EF-Tu and accordingly both species become more resistant to a range of phytopathogenic bacteria (Lacombe et al., 2010). This is a dramatic proof-of-concept for the ability to engineer broad-spectrum pest resistance in crop plants, and therefore emerges as a opportunity area for the future.

Relevant to all three areas of study discussed above is a fundamental understanding of how plant phenotype is controlled by genotype and environmental interactions (so called $\mathrm{G} \times \mathrm{E}$ interactions). It is critical that we be able to associate genotype with phenotype under constant environmental conditions, but also in response to variable conditions that approximate what plants experience in the field (including extremes). Genotyping is relatively fast compared to phenotyping, which is inherently much more difficult; e.g., what conditions to test, and what should be measured? There is a need for automated systems and non-destructive sampling and analysis of relevant plant growth parameters, and is an area of active work. For example, an iPlant Collaborative (Goff et al., 2011) has been established to address the genotype to phenotype problem, and the importance of developing high throughput phenotyping to match our genotyping capability is well recognized (Sinha, 2011).

These are only a few of the specific opportunities in the broad field of plant physiology that will likely lead to impacts on science and also societal needs, and thus emerge as challenges for multidisciplinary studies in the years to come. It has been noted in the past that, in general, we have the tools of molecular engineering and already know much about the molecular basis of growth and development and adaptation to the changes in the environment (including stress). However, it has proven difficult to apply this knowledge to improve crop productivity (Van Montagu, 2011), and to generate crops with increased stress tolerance (Mittler and Blumwald, 2010). Achieving this will require a multi-disciplinary approach with an underpinning provided by plant physiology.

\section{REFERENCES}

Barratt, D. H., Derbyshire, P., Findlay, K., Pike, M., Wellner, N., Lunn, J., Feil, R., Simpson, C., Maule, A. J., and Smith, A. M. (2009). Normal growth of Arabidopsis requires cytosolic invertase but not sucrose synthase. Proc. Natl. Acad. Sci. U.S.A. 106, 13124-13129.

Berger, B., Parent, B., and Tester, M. (2010). Highthroughput shoot imaging to study drought responses. J. Exp. Bot. 61, 3519-3528.

Blankenship, R. E., Tiede, D. M., Barber, J., Brudvig, G. W., Fleming, G., Ghirardi, M., Gunner, M. R., Junge, W., Kramer, D. M., Melis, A., Moore, T. A., Moser, C. C., Nocera, D. G., Nozik, A. J., Ort, D. R., Parson, W. W., Prince, R. C., and Sayre, R. T. (2011). Comparing photosynthetic and photovoltaic efficiencies and recognizing the potential for improvement. Science 332, 805-809.

Boothe, J., Nykiforuk, C., Shen, Y., Zaplachinski, S., Szarka, S., Kuhlman, P., Murray, E., Morck, D., and Moloney, M. M. (2010). Seed-based expression systems for plant molecular farming. Plant Biotechnol. J. 8, 588-606.

Charpentier, M., and Oldroyd, G. (2010). How close are we to nitrogen-fixing cereals? Curr. Opin. Plant Biol. $13,556-564$.

Chrispeels, M. J., and Raikhel, N. V. (2000). New initiatives to sustain our forward momentum. Plant Physiol. $122,1-2$.

Finkemeier, I., Laxa, M., Miguet, L., Howden, A. J., and Sweetlove, L. J. (2011). Proteins of diverse function and subcellular location are lysine acetylated in Arabidopsis. Plant Physiol. 155, 1779-1790.

Fu, C., Mielenz, J. R., Xiao, X., Ge, Y., Hamilton, C. Y., Rodriguez, M., Chen, F., Foston, M., Ragauskas, A. Bouton, J., Dixon, R. A., and Wang, Z.-Y. (2011). Genetic manipulation of lignin reduces recalcitrance and improves ethanol production from switchgrass. Proc. Natl. Acad. Sci. U.S.A. 108, 3803-3808.

Glozak, M. A., Sengupta, N., Zhang, X., and Seto, E. (2005). Acetylation and deacetylation of non-histone proteins. Gene 363, 15-23.

Goff, S. A., Vaughn, M., Mckay, S., Lyons, E., Stapleton, A. E., Gessler, D., Matasci, N., Wang, L., Hanlon, M., Lenards, A., Muir, A., Merchant, N., Lowry, S., Mock, S., Helmke, M., Kubach, A., Narro, M., Hopkins, N., Micklos, D., Hilgert, U., Gonzales, M., Jordan, C. Skidmore, E., Dooley, R., Cazes, J., Mclay, R., Lu, Z., Pasternak, S., Koesterke, L., Piel, W. H., Grene, R., Noutsos, C., Gendler, K., Feng, X., Tang, C., Lent, M., Kim, S.-J., Kvilekval, K., Manjunath, B. S., Tannen, V., Stamatakis, A., Sanderson, M., Welch, S. M., Cranston, K., Soltis, P., Soltis, D., O'meara, B., Ane, C., Brutnell, T., Kleibenstein, D. J., White, J. W., Leebens-Mack, J., Donoghue, M. J., Spalding, E. P., Vision, T. J., Myers, C. R., Lowenthal, D., Enquist, B. J., Boyle, B., Akoglu, A., Andrews, G., Ram, S., Ware, D., Stein, L., and Stanzione, D. (2011). The iPlant collaborative: cyberinfrastructure for plant biology. Front. Plant Sci. 2:34. doi: 10.3389/fpls.2011.00034

Hardin, S. C., Larue, C. T., Oh, M. H., Jain, V., and Huber, S. C. (2009). Coupling oxidative signals to protein phosphorylation via methionine oxidation in Arabidopsis. Biochem. J. 422, 305-312.

Haseloff, J., and Ajioka, J. (2009). Synthetic biology: history, challenges and prospects. J. R. Soc. Interface 6(Suppl. 4), S389-S391.

Heazlewood, J. L. (2011). Grand challenges in plant proteomics. Front. Plant Sci. 2:6. doi: 10.3389/ fpls.2011.00006 
Hibberd, J. M., and Covshoff, S. (2010). The regulation of gene expression required for C-4 photosynthesis. Annu. Rev. Plant Biol. 61, 181-207.

Hibberd, J. M., Sheehy, J. E., and Langdale, J. A. (2008). Using C4 photosynthesis to increase the yield of rice rationale and feasibility. Curr. Opin. Plant Biol. 11, 228-231.

Himmel, M. E., Ding, S.-Y., Johnson, D. K., Adney, W. S., Nimlos, M. R., Brady, J. W., and Foust, T. D. (2007). Biomass recalcitrance: engineering plants and enzymes for biofuels production. Science 315 , 804-807.

Kim, S. C., Sprung, R., Chen, Y., Xu, Y., Ball, H., Pei, J., Cheng, T., Kho, Y., Xiao, H., Xiao, L., Grishin, N. V., White, M., Yang, X. J., and Zhao, Y. (2006). Substrate and functional diversity of lysine acetylation revealed by a proteomics survey. Mol. Cell 23, 607-618.

Kim, T. W., and Wang, Z.Y. (2010). Brassinosteroid signal transduction from receptor kinases to transcription factors. Annu. Rev. Plant Biol. 61, 681-704.

Koerner, C. (2011). The grand challenges in functional plant ecology. Front. Plant Sci. 2:1. doi: 10.3389/ fpls.2011.00001

Lacombe, S., Rougon-Cardoso, A., Sherwood, E., Peeters, N., Dahlbeck, D., Van Esse, H. P., Smoker, M., Rallapalli, G., Thomma, B. P. H. J., Staskawicz, B., Jones, J. D. G., and Zipfel, C. (2010). Interfamily transfer of a plant pattern-recognition receptor confers broad-spectrum bacterial resistance. Nat. Biotech. $28,365-369$.

Long, S. P., Ainsworth, E. A., Leakey, A. D., Nosberger, J., and Ort, D. R. (2006). Food for thought: lowerthan-expected crop yield stimulation with rising $\mathrm{CO} 2$ concentrations. Science 312, 1918-1921.

Millar, A. H., Whelan, J., Soole, K. L., and Day, D. A. (2011). Organization and regulation of mitochondrial respiration in plants. Annu. Rev. Plant Biol. 62, 79-104.

Mittler, R., and Blumwald,E. (2010). Genetic engineering for modern agriculture: challenges and perspectives. Annu. Rev. Plant Biol. 61, 443-462.
Morinaka, Y., Sakamoto, T., Inukai, Y., Agetsuma, M., Kitano, H., Ashikari, M., and Matsuoka, M. (2006). Morphological alteration caused by brassinosteroid insensitivity increases the biomass and grain production of rice. Plant Physiol. 141, 924-931.

Mukhopadhyay, D., and Riezman, H. (2007). Proteasomeindependent functions of ubiquitin in endocytosis and signaling. Science 315, 201-205.

NRC. (2009). A New Biology for the 21st Century: Ensuring the United States Leads the Coming Biology Revolution. Washington, DC: National Academies Press.

Oh, M.-H., Sun, J., Oh, D.-H., Zielinski, R. E., Clouse, S. D., and Huber, S. C. (2011). Enhancing Arabidopsis leaf growth by engineering the BRASSINOSTEROID INSENSITIVE 1 receptor kinase. Plant Physiol. doi: 10.1104/pp.111.182741. [Epub ahead of print].

Popper, Z. A., Michel, G., Hervé, C., Domozych, D. S., Willats, W. G. T., Tuohy, M. G., Kloareg, B., and Stengel, D. B. (2011). Evolution and diversity of plant cell walls: from algae to flowering plants. Annu. Rev. Plant Biol. 62, 567-590.

Rizhsky, L., Liang, H., Shuman, J., Shulaev, V., Davletova, S., and Mittler, R. (2004). When defense pathways collide. The response of Arabidopsis to a combination of drought and heat stress. Plant Physiol. 134, 1683-1696.

Schmidt, M. A., and Herman, E. M. (2008). Proteome rebalancing in soybean seeds can be exploited to enhance foreign protein accumulation. Plant Biotechnol. J. 6, 832-842.

Sinha, N. R. (2011). Plant developmental biology in the post-genomic era. Front. Plant Sci. 2:11. doi: 10.3389/ fpls.2011.00011

Somers, D. E., and Fujiwara, S. (2009). Thinking outside the F-box: novel ligands for novel receptors. Trends Plant Sci. 14, 206-213.

Sugiyama,N.,Nakagami,H.,Mochida,K., Daudi,A., Tomita M., Shirasu, K., and Ishihama, Y. (2008). Large-scale phosphorylation mapping reveals the extent of tyrosine phosphorylation in Arabidopsis. Mol. Syst. Biol. 4, 193.

Uhrig, R. G., She, Y. M., Leach, C. A., and Plaxton, W. C. (2008). Regulatory monoubiquitination of phospho- enolpyruvate carboxylase in germinating castor oil seeds. J. Biol. Chem. 283, 29650-29657.

Van Montagu, M. (2011). It is a long way to GM agriculture. Annu. Rev. Plant Biol. 62, 1-23.

Varshney, R. K., Bansal, K. C., Aggarwal, P. K., Datta, S. K., and Craufurd, P. Q. (2011). Agricultural biotechnology for crop improvement in a variable climate: hope or hype? Trends Plant Sci. 16, 363-371.

Wang, Q., Zhang, Y., Yang, C., Xiong, H., Lin, Y., Yao, J., Li, H., Xie, L., Zhao, W., Yao, Y., Ning, Z. B., Zeng, R., Xiong, Y., Guan, K. L., Zhao, S., and Zhao, G. P. (2010). Acetylation of metabolic enzymes coordinates carbon source utilization and metabolic flux. Science 327, 1004-1007.

Wu, X., Oh, M. H., Schwarz, E. M., Larue, C. T., Sivaguru, M., Imai, B. S., Yau, P. M., Ort, D. R., and Huber, S. C. (2011). Lysine acetylation is a widespread protein modification for diverse proteins in Arabidopsis. Plant Physiol. 155, 1769-1778.

Zavala, J.A., Casteel, C. L., Delucia, E. H., and Berenbaum, M. R. (2008). Anthropogenic increase in carbon dioxide compromises plant defense against invasive insects. Proc. Natl. Acad. Sci. U.S.A. 105, 5129-5133.

Zwieniecki, M. A., and Dumais, J. (2011). Quantifying green life: grand challenges in plant biophysics and modeling. Front. Plant Sci. 2:31. doi: 10.3389/ fpls.2011.00031

Received: 03 August 2011; accepted: 22 August 2011; published online: 08 September 2011.

Citation: Huber SC (2011) Grand challenges in plant physiology: the underpinning of translational research. Front. Physio. 2:48. doi: 10.3389/fpls.2011.00048

This article was submitted to Frontiers in Plant Physiology, a specialty of Frontiers in Plant Science.

Copyright (ㅇ) 2011 Huber. This is an open-access article subject to a non-exclusive license between the authors and Frontiers Media SA, which permits use, distribution and reproduction in other forums, provided the original authors and source are credited and other Frontiers conditions are complied with. 